\title{
Application of Capillary Network Air Conditioning System Based on Three-constant Technology in Gymnasium
}

\author{
Yuhua Yang ${ }^{1, *}$, Fan Zhang ${ }^{2}$ \\ ${ }^{1}$ Beijing University of Chemical Technology,Logistics Support Department, Beijing, China \\ ${ }^{2}$ Beijing Jiaotong University,Infrastructure and Planning Department, Beijing, China
}

\begin{abstract}
With the advancement of social development, the continuous improvement of the level of science and technology puts higher requirements on the environmental quality of modern large-scale venues, which requires innovative concepts and high-tech to meet the requirements. In the paper, the capillary radiant air conditioning system based on three-constant technology is taken as the research object, and the steady heat transfer mathematical model of heat transfer from the cold water in the capillary network to the room is established. The structural parameters and operating parameters of the capillary radiant air conditioner are simulated and analyzed for the influence of its thermal performance, the environmental quality detection and control method based on bilinear model was established, and the algorithm of each index parameter was optimized to improve the high consistency between the model prediction parameters and the control tracking parameters. After verifying and testing the model parameters, it proves that the research content of the paper is real and effective, which its effect has reached the value of practical application. Through the research on the capillary network air conditioning system based on three-constant technology, it proves that the application in the construction of large venues has the effect of energy saving and environmental protection, and provides an innovative reference for the construction and development of the stadium.
\end{abstract}

\section{Introduction}

With the continuous development of social economy, the requirements for indoor environment thermal comfort in large-scale stadium construction are also constantly improving. Although traditional air conditioners can meet the environmental requirements of venues, there are some problems, such as loss caused by temperature and humidity coupling processing, cold surface breeding mold, convection blowing, coil air supply noise and indoor installation of two sets of environmental conditioning systems; and due to technical problems caused by the air conditioning system principle structure cannot completely solve the above problems, but also improve The degree of comfort inevitably leads to greater energy loss and also creates a serious problem of energy conservation. In the traditional air conditioning, air convection heat is used to control the temperature of the air, and the airflow of a certain wind speed also takes away the heat of the human body to cause discomfort. Even in the summer, the cold air blowing the human body is not good for the health of the body. Based on the drawbacks of the traditional air conditioning operating principle, the innovative design of the capillary network air conditioning system in principle uses the form of radiative heat transfer to completely avoid these problems[1-2].

Three-constant technology includes radiation heating or refrigeration, fresh air humidity control, air purification, intelligent control and other functions. Taking the whole year as a whole, Fitzgerald three-constant technology system is designed according to bionics principle, which comprehensively solves the needs of temperature, humidity, oxygen content, cleanliness and low noise in the environment. Capillary radiation air conditioning is widely used as a form of air conditioning to create a comfortable environment in space. Combining with three-constant technology, it can improve the comfort of indoor temperature, humidity and oxygen content, and solve the problem that the structure parameters and operation conditions of capillary radiation air conditioning affect its cooling capacity.

The capillary network air conditioning system is based on the independent control technology of temperature and humidity and the principle of long-wave radiation. Combined with displacement ventilation, it can effectively improve the indoor air quality and realize the cascade utilization of energy, and is a comfortable, energy-saving and environmentally-friendly air-conditioning system. Capillary radiant air conditioners alone need to be combined with other cooling methods due to insufficient cooling, and their structural parameters and operating conditions directly affect their cooling capacity. The air conditioning system incorporating three-constant technology will greatly reduce the system operating power consumption, which realizes the practical application of capillary radiant air conditioning in the construction of large venues. It not only becomes the best 
solution for air-conditioning systems for large-scale construction projects, but also guides the way for innovative green building construction [3-4].

The rise of three-constant system is based on people's requirements for the quality of space air environment, which aims to make people enjoy the high-quality healthy life, the fresh air and the healthy environment with appropriate temperature and humidity have attracted a lot of attention.

The application of three-constant technology in capillary network air conditioning system based on temperature and humidity independent control technology and long-wave radiation principle, combined with displacement ventilation can effectively improve the air quality in the stadium and realize the cascade utilization of energy, is a comfortable, energy-saving, environmentally friendly air conditioner system [5]. In the paper, the heat transfer physical model of the capillary micro-environment is first established, and the heat transfer analysis of the whole air conditioning system is carried out. The numerical simulation is carried out by using CFD software to explore the temperature distribution of the capillary tube and the influence of heat exchange capacity with different tube spacing, plastering material and temperature difference of supply and return water; secondly, the physical model of the ceiling-type capillary radiant end air-conditioned room was established, and the CFD software was used for numerical simulation to obtain the distribution of temperature field and velocity field in the cooling room under different roof temperature, air supply mode and supply air temperature. At the same time, Matlab software is used to program the PMV-PPD evaluation index equation to obtain the thermal comfort of the human body in the radiant air conditioning system. Finally, the application of the capillary network air conditioning system in the gym is used for experimental verification, and the temperature field, velocity field and relative The humidity was tested and the test data was analyzed and compared with the numerical simulation results above. The experimental results show that the measured values are basically consistent with the numerical simulation values, which proves that it is feasible to use the numerical simulation method to predict the thermal environment of the capillary network air conditioning system. The calculation results show that the pipe spacing, plastering material, water supply flow rate and temperature have a significant influence on the heat transfer performance of the roof. The optimal parameter value can ensure the strongest heat exchange capacity of the roof.

\section{2 capillary Network Air Conditioning system based on Three-Constant Technology}

In the paper, the capillary radiant air conditioning system used in the construction of stadiums is taken as the research object. By establishing the steady heat transfer mathematical model of heat transfer from the cold water in the capillary network to the room, the effects of structural parameters and operating parameters of capillary radiant air conditioning on its thermal performance are simulated and analyzed. The results show that changing the structural parameters, such as the use of tubes with high thermal conductivity and laying layer materials, increasing the capillary diameter or reducing the tube spacing, can improve the operating efficiency of the air-conditioning system in the stadium, reduce system energy consumption and greatly improve the extension of the system's service life [6].

According to the PMV standard of thermal comfort index, the threshold is set to $-0.5-+0.5$ is the best, which the predicted percentage dissatisfied (PPD) is within 10\%, and the result shows that the air supply mode is sent back and forth. It is superior to other air supply methods; the top plate temperature is the main factor affecting the thermal comfort of the human body, and the predicted dissatisfaction rate is less than $10 \%$ when the temperature is not more than $20^{\circ} \mathrm{C}$. In the control logic structure of the capillary network air conditioning system, it is mainly to effectively control the continuous change of environmental parameters, and to regularly manage the classification of various air indicators in the rules governing air quality, which achieve the purpose of micro-control management. The operation process of the main program of the system is shown in Figure 1.

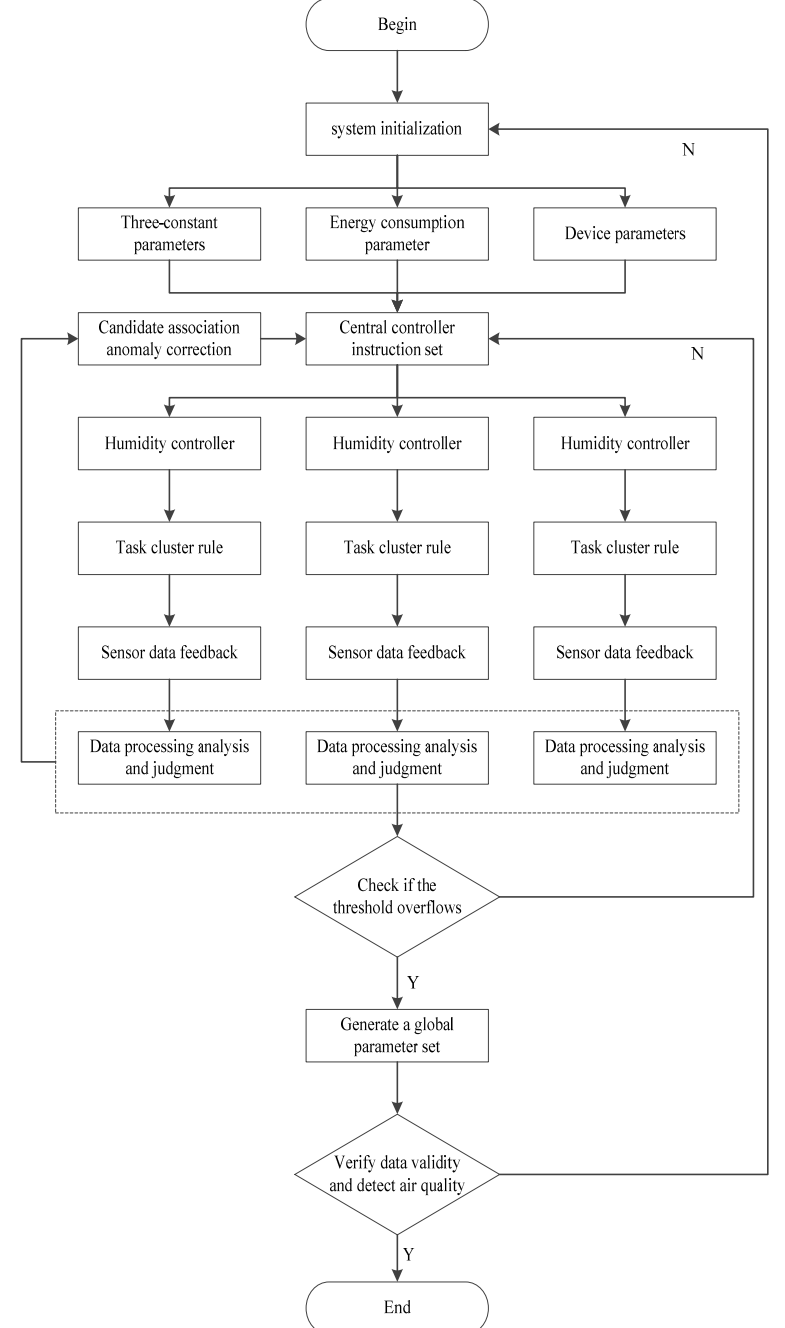

Figure 1. Capillary network air conditioning system program operation process. 


\section{Gymnasium Capillary Network Air Conditioning System Control Model}

The stadium capillary network air conditioning control system includes the system controller, the three-constant state actuator, the energy management system and various information acquisition modules. Among them, ACT is an actuator that improves the environmental quality of the venue that includes air conditioning equipment and exhaust fans. BEMS is a building energy management system that collects environmental quality parameters and controls the actuators in the venue. $\mathrm{N}$ is a sample of the energy management system, and the structural model is shown in Figure 2.

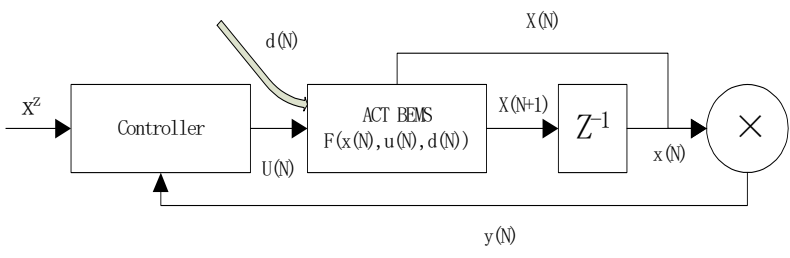

Figure 2. Control model of gymnasium capillary network air conditioning system.

Where, $x(N)$ is the state variable, $y(N)$ is the reference vector, $d(N)$ is the disturbance, and $X^{z}$ is the comfort setting vector.

The environmental quality control system based on the model in the stadium can be described as the following equation; its state equation is as follows.

$$
x(N+1)=f[x(N), u(N), d(N)]
$$

Reference vector is

$$
y(N)=x(N)
$$

Control vector is

$$
\begin{aligned}
& \qquad u(N)=g[x(z), y(N)] \\
& \qquad O_{2(\text { in })}(N+1)=c O_{2(\text { in })}(N)+a w \\
& \text { Where } \alpha \text { is the model parameter that needs to be } \\
& \text { identified. The temperature in the venue at time } N+1 \text { is } \\
& \text { the temperature inside and outside the venue at time } N \text {. } \\
& \text { The linear function of the ventilation of exhaust fan } \\
& \text { with the cooling and heating of air conditioner is defined } \\
& \text { as follows. } \\
& \mathrm{T}_{\text {in }}(N+1)=T_{\text {in }}(N)+\beta_{1}\left[T_{\text {out }}(N)-T_{\text {in }}(N)\right]+\beta_{2} a(N) \\
& +\beta_{3} w(N)\left[T_{\text {out }}(N)-T_{\text {in }}(N)\right]
\end{aligned}
$$

Where, $\beta_{1}, \beta_{2}$, and $\beta_{3}$ are model parameters that need to be identified.

The model of the control system based on model prediction can be expressed as follows.

$$
x(N+1)=B x(N)+C x(N) w(N)+F u(N)
$$

Where,

$$
B=\left[\begin{array}{cccc}
1 & 0 & 0 & 0 \\
0 & 1-\beta_{1} & 0 & \beta_{1} \\
0 & 0 & \phi_{1} & 0 \\
0 & 0 & 0 & \phi_{2}
\end{array}\right]
$$

According to the degree of response of the stadium environment to the air conditioning identification system, the bilinear model is used for air quality management regardless of the presence of noise.

$$
\begin{aligned}
& x(N+1)=x(N)+\rho_{1} f_{1}[x(N), u(N), d(N)]+\rho_{2} f_{2}[x(N), u(N), d(N)]+ \\
& \cdots \rho_{t} f_{t}[x(N), u(N), d(N)]
\end{aligned}
$$

Where $\rho_{i}=\rho_{1}, \rho_{2}, \cdots \rho_{t}$ is the parameter to be identified in the model, and the state vector $x(N)$ is defined as follows.

$$
x(N)=\left[\mathrm{co}_{2(\text { in })}(N), T_{\text {in }}(N), \mathrm{co}_{2(\text { out })}(N), T_{\text {out }}(N)\right]
$$

In the (5), $\mathrm{CO}_{2(i n)}$ is the concentration of carbon dioxide in the venue at time $N, T_{i n}(N)$ is the temperature in the venue at time $\mathrm{N}, \mathrm{CO}_{2(\text { out })}(\mathrm{N})$ is the outdoor carbon dioxide concentration at time $N$, and $T_{i n}(N)$ is the outdoor temperature at time $N$. The control vector is defined as follows.

$$
u(N)=[w(N), A(N)]
$$

In the $(5), w(N)$ is the ventilation amount of the exhaust fan at the time $N$, and $A(N)$ is the cooling and heating capacity of the air conditioner at the time $N$. The comfort setting vector $x^{z}$ is defined as $x^{z}=\left[\mathrm{Co}_{2(z)}, T_{z}\right], \mathrm{CO}_{2(z)}$, which is the carbon dioxide concentration in the set venue, and $T_{z}$ is the set temperature in the venue.

For the carbon dioxide and temperature state variables in the venue, the bilinear model is described in different forms. The carbon dioxide concentration in the venue at time $N+1$ is a linear function of the concentration of carbon dioxide in the venue at time $N$ and the ventilation of the exhaust fan, and the function is defined as follows.

$$
\begin{gathered}
C=\left[\begin{array}{cccc}
-a & 0 & a & 0 \\
0 & 1-\beta_{3} & 0 & \beta_{3} \\
0 & 0 & 0 & 0 \\
0 & 0 & 0 & 0
\end{array}\right] \\
\mathrm{F}=\left[\begin{array}{cc}
0 & 0 \\
0 & \beta_{2} \\
0 & 0 \\
0 & 0
\end{array}\right]
\end{gathered}
$$

In the (12), $\phi_{1}$ and $\phi_{2}$ are the relationship between the previous moment and the next moment of the carbon dioxide concentration and temperature parameters in the outdoor climate, they can be obtained by measurement.

\section{Test Verification and Data Analysis}

The test environment of this paper is established in Hengxin Swimming Hall of Kaifeng City, Henan Province. The venue area is $65,000 \mathrm{~m}^{2}$, and the venue is 
$25 \mathrm{~m}$ high. The outer surface is a concrete wall with a thickness of $35 \mathrm{~mm}$. The other two sides are glass partition walls with a thickness of $5 \mathrm{~mm}$. The top is a concrete slab with a thickness of $30 \mathrm{~mm}$.

The energy management system uses DeYiAn building energy management and control platform to collect indoor environmental parameters through indoor temperature and carbon dioxide concentration sensors. Considering the position of the sensor will affect the measurement results, four temperature sensors are used in the experiment and four $\mathrm{CO}_{2}$ concentration sensors, which are arranged at four corners of the room that about $1.6 \mathrm{~m}$ away from the ground. We use the adaptive weighting method to fuse the values of the four sensors, and use the fusion result as the basis for final control. The outdoor data is based on Crossbow's weather station system provides data. Both air conditioners and exhaust fans are connected to the platform to control the temperature and $\mathrm{CO}_{2}$ concentration in the room via infrared remote control. The complexity of air conditioning and exhaust fan control is simplified during the experiment. For air conditioning control, the air volume is unchanged, set to medium speed, adjust the set temperature of the air conditioner, from $20^{\circ} \mathrm{C}$ to $28^{\circ} \mathrm{C}$, corresponding to the energy consumption management and control platform air conditioning opening control signal control accuracy is $0.5^{\circ} \mathrm{C}$; For fan control is to adjust the air volume of the fan, from 0 to $5000 \mathrm{~m}^{3}$, the control accuracy is $400 \mathrm{~m}^{3}$ per hour.

In order to obtain real experimental data, the energy management and control platform is set to a continuous working mode for the outdoor weather conditions in Kaifeng City in summer. The number of indoor personnel is basically maintained at 600 , and the control system controls the air conditioner and exhaust fan according to the step of $10 \%$. The cooling, heating and exhausting are cycled within $24 \mathrm{~h}$. The control signal is transmitted to the infrared remote control through the building energy management and control platform, and the operation of the air conditioner and the fan is controlled by the infrared remote controller. The indoor environmental parameters and the control amount of the air conditioner and the exhaust fan are observed, and the outdoor temperature is recorded and sample data as the identified sample data. The sampling point data of the air conditioning system is shown in Table 1.

Table 1. Gymnasium Air Temperature Sampling Statistics

\begin{tabular}{|l|l|l|l|l|l|l|l|}
\hline $\begin{array}{c}\text { Temperature/ } \\
{ }^{\circ} \mathrm{C}\end{array}$ & $\mathbf{1 0} \mathbf{~ m i n}$ & $\mathbf{2 0} \mathbf{m i n}$ & $\mathbf{3 0} \mathbf{~} \mathbf{m}$ & $\mathbf{4 0} \mathbf{~ m i n}$ & $\mathbf{5 0} \mathbf{~ m i n}$ & $\mathbf{6 0} \mathbf{~ m i n}$ & $\ldots$ \\
\hline coordinate a & 26.7 & 27.6 & 27.4 & 27.3 & 26.9 & 26.8 & $\ldots$ \\
\hline coordinate b & 26.9 & 27.4 & 27.5 & 26.9 & 27.2 & 27.4 & $\ldots$ \\
\hline coordinate c & 26.9 & 27.1 & 27.7 & 27.3 & 27.6 & 27.3 & $\ldots$ \\
\hline coordinate d & 27.2 & 27.5 & 27.5 & 27.3 & 27.4 & 27.3 & $\ldots$ \\
\hline coordinate e & 26.8 & 26.9 & 27.4 & 27.7 & 27.8 & 27.3 & $\ldots$ \\
\hline coordinate f & 26.8 & 26.4 & 27.5 & 27.3 & 27.2 & 27.6 & $\ldots$ \\
\hline coordinate g & 26.9 & 26.5 & 27.3 & 27.3 & 27.6 & 27.8 & $\ldots$ \\
\hline
\end{tabular}

The data in Table I is used as a temperature sampling standard for data statistics, and the sampling data statistics of humidity, carbon dioxide concentration and air volume are also performed. According to the analysis of the power consumption and efficiency ratio of the air conditioning system, as shown in Figure 3, the capillary network air conditioning system in the swimming pool controls the effect.

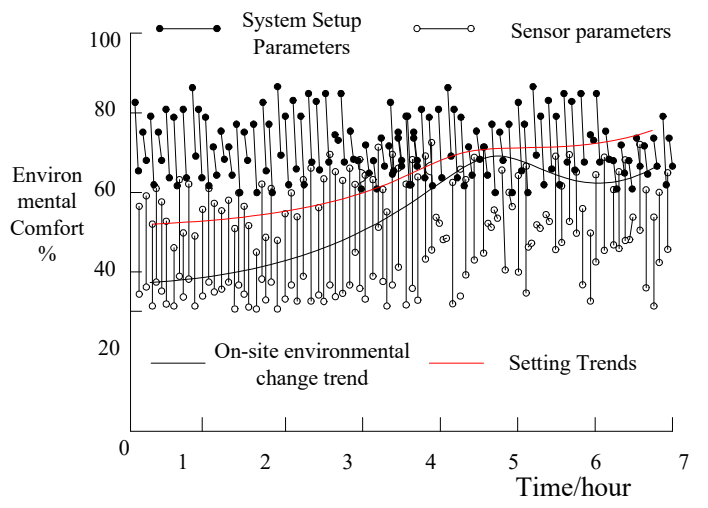

Figure 3. Analysis of air conditioning control effect in swimming pool capillary network.

In Figure 3, the actual situation of each environmental detection sensor is compared according to the capillary network air conditioning system in the swimming pool. The data analyzed the difference between the actual air environment and the system setting parameters in the swimming pool, and made a linear comparison based on the environmental indicators of the three constant parameters. According to the intervention time of the air conditioning system, the system setting index and the actual environmental variable are within the range of $\pm 7.5 \%$ of the threshold setting. As time changes, the trend value gradually decreases during the continuous learning process, and the system setting value becomes more and more close to environmental variables. Experiments of capillary gravity circulation cooling mode and central air conditioning cooling capacity are carried out in the outdoor temperature range where the maximum cooling load occurs. The experimental results are shown in Figure 4. Although the outdoor temperature is in the maximum cooling load range, the capillary gravity circulation cooling mode and the central air conditioning cooling mode can maintain the indoor design temperature. Each cooling mode can meet the stability and maintenance of the indoor design temperature. Therefore, the cooling capacity of the capillary gravity circulation cooling mode can meet the summer cooling load of residential buildings. 


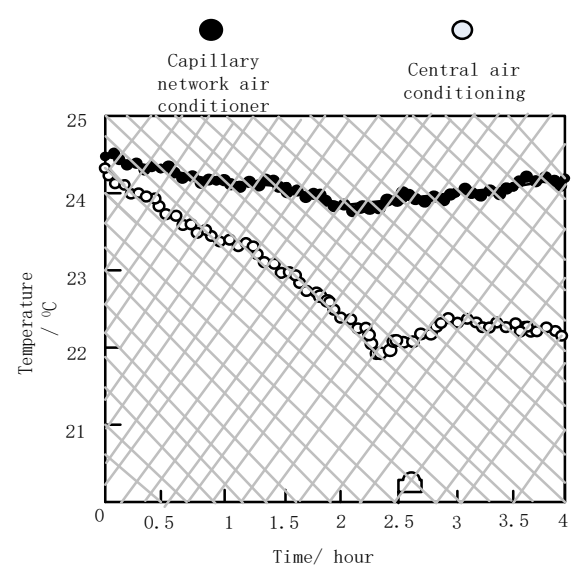

Figure 4. Comparison of outdoor cooling load test results.

\section{Conclusions}

In the paper, capillary air-conditioning system is taken as the research object, and the reasonable value of capillary network structure and operating parameters based on three-constant technology is discussed. By establishing the environmental quality detection and control method with bilinear model, and optimizing the algorithm of each index parameter, the high consistency of the model prediction parameters and the control tracking parameters is improved. Based on model predictive control and optimization, the regulation of indoor environmental quality ensures good indoor environmental quality and meets the requirements of lower energy consumption. At the same time, control and optimization methods have a fast and stable response to outdoor climate change. The test results show that the system not only has the effect of saving energy, but also can meet the needs of large-scale stadium construction, and has a good market prospect for the future construction industry.

\section{References}

1. G. N. Li, Y. P. Hu, H. X. Chen, L. M. Shen, H. R. Li, J. Li and W. J. Hu. (2016)Extending the virtual refrigerant charge sensor(VRC) for variable refrigerant flow (VRF) air conditioning system using data-based analysis methods, Applied Thermal Engineering, vol. 93, 908-919.

2. Y. Zhao, J. Wen and S. W. Wang. (2015) Diagnostic Bayesian networks for diagnosing air handling units faults-Part II: Faults incoils and sensors, Applied Thermal Engineering, vol. 90, 145-157.

3. T. Mulumba, A. Afshari, K. Yan, W. Shen, L. K. Norford. (2015)Robust model-based fault diagnosis for air handling units, Energy and Buildings, vol. 86, 698-707.

4. Y. Y. Tzou. R. S. Ou, S. L. Jung and M. Y. Chang.(1997) High-performance Programmable AC Power Source with Low Harmonic Distortion Using DSP-based Repetitive Control Technique, IEEE Transaction on Power Electronics, vol. 12, no. 4, 715-725.
5. Chihchiang Hua. (1995) Two-level Switching Pattern Deadbeat DSP Controlled PWM inverter, IEEE Transaction on Power Electronics, vol. 10 ,no. 3, 310-317.

6. Y. F. Fan and P. Li. (2002) PID Parameters Setting up, China Instrumentation, vol. 128, no. 3, 24-28. 